\title{
HNCO enhancement by shocks in the L1157 molecular outflow
}

\author{
N. J. Rodríguez-Fernández ${ }^{1}$, M. Tafalla ${ }^{2}$, F. Gueth ${ }^{1}$, and R. Bachiller ${ }^{2}$ \\ 1 IRAM, 300 rue de la Piscine, 38406 St. Martin d'Heres, France \\ e-mail: rodriguez@iram.fr \\ 2 Observatorio Astronomico Nacional, IGN, calle Alfonso XII 3, 28014 Madrid, Spain
}

Received 5 January 2010 / Accepted 23 March 2010

\section{ABSTRACT}

\begin{abstract}
Context. The isocyanic acid (HNCO) has an extended distribution in the centers of the Milky Way and the spiral galaxy IC342. Based on both the morphology of the emission and the $\mathrm{HNCO}$ abundance with respect to $\mathrm{H}_{2}$, several authors hypothesized that $\mathrm{HNCO}$ could be a good tracer of interstellar shocks.

Aims. We test this hypothesis by observing a well-known Galactic source whose chemistry is dominated by shocks.

Methods. We observed several transitions of HNCO towards L1157-mm and two positions (B1 and B2) in the blue lobe of the molecular outflow.

Results. The HNCO line profiles exhibit the same characteristics as other well-known shock tracers such as $\mathrm{CH}_{3} \mathrm{OH}, \mathrm{H}_{2} \mathrm{CO}, \mathrm{SO}$, or $\mathrm{SO}_{2}$. The three molecules $\mathrm{HNCO}, \mathrm{SO}_{2}$, and OCS are the only ones detected so far whose emission is more intense in $\mathrm{B} 2$ than in $\mathrm{B} 1$, making these species valuable probes of chemical differences along the outflow. The $\mathrm{HNCO}$ abundance with respect to $\mathrm{H}_{2}$ is $(0.4-1.8) \times 10^{-8}$ in B1 and $(0.3-1) \times 10^{-7}$ in B2. These abundances are the highest ever measured, and imply an increment with respect to L1157-mm of up to a factor of 83 , demonstrating that this molecule is a good shock tracer.

Conclusions. we demonstrate that shocks can produce the HNCO abundance measured in galactic nuclei and even higher values. We propose that the gas phase abundance of HNCO is produced by both grain mantle erosion by the shock waves and by neutral-neutral reactions in gas phase involving $\mathrm{CN}$ and $\mathrm{O}_{2}$. The observed anticorrelation between $\mathrm{CN}$ and HNCO fluxes supports this scenario. The observed similarities between the HNCO emission and sulfur-bearing molecules may be caused by formation pathways that also involve $\mathrm{O}_{2}$.
\end{abstract}

Key words. ISM: individual objects: L1157 - ISM: jets and outflows - ISM: molecules - stars: formation

\section{Introduction}

Interstellar isocyanic acid (HNCO) was first detected towards Sgr B2 (Snyder \& Buhl 1972; Churchwell et al. 1986; Kuan \& Snyder 1996). Since the first detection in this source, the molecule has been observed in other hot cores around massive (Blake et al. 1987; MacDonald et al. 1996) and low mass protostars (van Dishoeck et al. 1995; Bisschop et al. 2008). It has also been detected in translucent clouds (Turner et al. 1999) and in the dense regions of Galactic molecular clouds (Jackson et al. 1984; Zinchenko et al. 2000), including those at the Galactic center (Hüttemeister 1993; Lindqvist et al. 1995; Dahmen et al. 1997; Rizzo et al. 2000; Minh et al. 2005; Martín et al. 2008). HNCO has also been detected in some extragalactic sources (Nguyen-QRieu et al. 1991; Meier \& Turner 2005; Martín et al. 2009). The isotopologue HCNO (fulminic acid) was detected by Marcelino et al. (2009).

The emission in HNCO lines with $K_{-1}=0^{1}$ is clearly extended in the two Galactic nuclei where it has been mapped: IC342 (Meier \& Turner 2005) and the Milky Way (Lindqvist et al. 1995; Dahmen et al. 1997). In the Galactic center, the spatial distribution of the $\mathrm{HNCO}$ emission differs from that of most other tracers. In particular, there is a HNCO peak without any associated $\mathrm{CO}$ peak at Galactic longitude $l=1.65^{\circ}$

1 HNCO is a planar, nearly linear, slightly asymmetric prolate rotor. The notation for a given level is $J_{K_{-1} K_{1}}$. The $K_{-1}=0$ are usually excited thermally, while the excited $K$ ladders $\left(K_{-1}>0\right)$ are probably excited by FIR radiation (Churchwell et al. 1986).
(Dahmen et al. 1997). This very special distribution suggests that HNCO may be an important tracer of some physical processes that are not well revealed by other molecules. It is possible that the molecule is tracing shocks, which are understood to occur at this Galactic longitude due to the gas dynamics in the barred potential of the Milky Way (Rodriguez-Fernandez \& Combes 2008). At the Galactic center, the highest gas phase abundances of $\mathrm{SiO}$ (a well-known shock tracer) are measured in this region (Hüttemeister et al. 1998; Rodriguez-Fernandez et al. 2006). Because of the special morphology of the HNCO emission in Sgr B2, Minh \& Irvine (2006) suggested that HNCO is enhanced by shocks. Regarding IC342, the HNCO emission resembles that of $\mathrm{CH}_{3} \mathrm{OH}$. In particular, $\mathrm{HNCO}$ is detected not only in the nuclear ring but also in the inner spiral arms or dustlanes. Since the dustlanes are understood to be the locus of strong shocks in barred galaxies (see for instance RodriguezFernandez \& Combes 2008, and references therein), Meier \& Turner (2005) suggested that $\mathrm{HNCO}$, as $\mathrm{CH}_{3} \mathrm{OH}$, could trace large-scale shocks.

Nevertheless, the hypothesis that HNCO is a good shock tracer on the scale of galaxies still needs to be probed since the HNCO emission has never been studied in well-known Galactic templates of interstellar shocks. To understand more clearly the excitation and the chemistry of this promising molecule, we observed the protostar L1157 and its associated molecular outflow. This outflow presents the morphological signature of shocks (Gueth et al. 1998; Codella et al. 2009) and is frequently used to benchmark numerical models of shocks (Gusdorf et al. 2008a,b). 


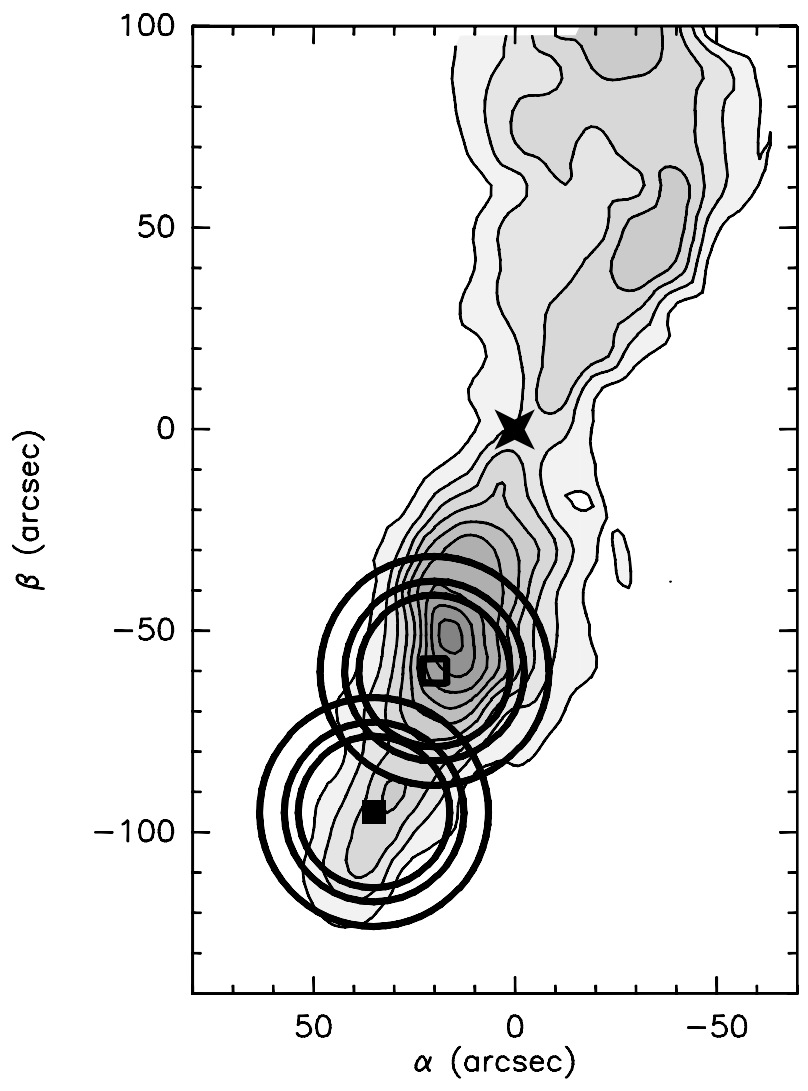

Fig. 1. Integrated CO(2-1) emission from the L1157 outflow (Bachiller et al. 2001). We show the position of L1157-mm (star), B1 (empty square), and B2 (filled square). B1 and B2 are the positions of the shocks as traced, for instance, by $\mathrm{SiO}(3-2)$ (see Fig. 6 of Bachiller et al. 2001). The CO emission peaks behind the true positions of the shocks. The different circles represent the half maximum contours of the IRAM $30 \mathrm{~m}$ beam at the frequencies of the lines discussed in this paper (see Table 1).

The L1157 outflow is the clearest example of "chemically active outflow" (Bachiller et al. 2001) and a template of shock chemistry, since many species have significantly higher abundances than the protostar (Bachiller \& Perez Gutierrez 1997). Therefore, it is a source of choice to characterize the emission of a given molecule in a shocked environment (see for instance Bachiller \& Perez Gutierrez 1997; Bachiller et al. 2001; Benedettini et al. 2007; Arce et al. 2008; Codella et al. 2009).

\section{Observations}

We observed three lines of the $K_{-1}=0$ ladder of HNCO towards the two main shocks in the southern lobe of the L1157 molecular outflow, the B1 and B2 positions of Bachiller \& Perez Gutierrez (1997). In addition, as reference we observed the continuum source L1157-mm (the protostar). The equatorial coordinates of L1157-mm are RA $=20^{\mathrm{h}} 39^{\mathrm{m}} 06.19^{\mathrm{s}}$, Dec $=68^{\circ} 02^{\prime} 15.9^{\prime \prime}$, (J2000). The offsets of B1 and B2 with respect to L1157-mm are $\left(20^{\prime \prime},-60^{\prime \prime}\right)$ and $\left(35^{\prime \prime},-95^{\prime \prime}\right)$, respectively.

The observations were performed with the IRAM $30 \mathrm{~m}$ telescope in Pico Veleta in July 2007. The line quantum numbers, frequencies, and the telescope parameters are listed in Table 1. As backends, we used the $100 \mathrm{KHz}$ filterbank and the VESPA autocorrelator with a channel resolution of $20-40 \mathrm{MHz}$, which allows us to study in detail the line wings. The observations were carried out in position-switching mode with the OFF position
Table 1. Observational parameters.

\begin{tabular}{ccccccc}
\hline \hline Line & $\begin{array}{c}v^{a} \\
\mathrm{GHz}\end{array}$ & $B_{\mathrm{eff}^{b}}$ & $F_{\mathrm{eff}^{c}}$ & $\begin{array}{c}F W H M^{d} \\
\operatorname{arcsec}\end{array}$ & $\begin{array}{c}T_{\text {sys }}{ }^{e} \\
\mathrm{~K}\end{array}$ & $\begin{array}{c}\mathrm{rms} \\
\mathrm{mK}\end{array}$ \\
\hline $4-3^{f}$ & 87.925 & 0.78 & 0.95 & 28.4 & 100 & $12-18$ \\
$5-4^{g}$ & 109.905 & 0.75 & 0.95 & 22.3 & 180 & $16-19$ \\
$6-5^{h}$ & 131.885 & 0.69 & 0.93 & 18.8 & 215 & $28-34$ \\
\hline
\end{tabular}

Notes. ${ }^{(a)}$ Frequency; ${ }^{(b)}$ beam efficiency; ${ }^{(c)}$ forward efficiency; ${ }^{(d)}$ full width at half maximum of the telescope main beam; ${ }^{\left({ }^{e}\right)}$ system temperature; ${ }^{(f)} 4_{0,4}-3_{0,3} ;{ }^{(g)} 5_{0,5}-4_{0,4} ;{ }^{(h)} 6_{0,6}-5_{0,5}$.

Table 2. Measured fluxes.

\begin{tabular}{lccc}
\hline \hline Line & $\begin{array}{c}\mathrm{mm}^{a} \\
\mathrm{~K} \mathrm{~km} \mathrm{~s}^{-1}\end{array}$ & $\begin{array}{c}\mathrm{B}^{b} \\
\mathrm{~K} \mathrm{~km} \mathrm{~s}^{-1}\end{array}$ & $\begin{array}{c}\mathrm{B}^{c} \\
\mathrm{Km} \mathrm{s}^{-1}\end{array}$ \\
\hline $4_{0,4}-3_{0,3}$ & 0.19 & 0.71 & 1.24 \\
$5_{0,5}-4_{0,4}$ & 0.19 & 0.84 & 1.38 \\
$6_{0,6}-5_{0,5}$ & 0.073 & $<0.25$ & $<0.20$ \\
\hline
\end{tabular}

Notes. Computed in the velocity range from: (a) 1.1 to $3.7 \mathrm{~km} \mathrm{~s}^{-1}$; (b) -4.8 to $5.0 \mathrm{~km} \mathrm{~s}^{-1}$; (c) -2.6 to $6.1 \mathrm{~km} \mathrm{~s}^{-1}$.

located 24' NE of L1157-mm. Typical system temperatures and rms noise of the spectra are given in Table 1.

Table 2 indicates the integrated flux of the different lines computed from spectra in $T_{\mathrm{mb}}$ units (forward and beam efficiencies are given in Table 1). The uncertainties in the calibration of the IRAM $30 \mathrm{~m}$ telescope are within $10 \%$ at the frequency of the lines discussed here (Mauersberger et al. 1989).

\section{Results}

\subsection{Line profiles: differences between the observed positions}

Figure 2 shows the spectra. The $4_{04}-3_{03}$ and $5_{05}-4_{04}$ lines were detected at all the positions. In addition the $6_{06}-5_{05}$ line was also detected at L $1157 \mathrm{~mm}$. The lines are narrow towards L1157-mm (linewidths of $0.7-0.9 \mathrm{~km} \mathrm{~s}^{-1}$ ) but the linewidth increases by more than a factor of 3 towards the B1 and B2 positions. In particular, they have prominent blue-wings towards B1.

Figure 3 compares of the HNCO $\left(5_{05}-4_{04}\right)$ line profile measured towards the three observed positions and the spectra of other molecules observed by Bachiller \& Perez Gutierrez (1997). The line profiles are in overall good agreement with the other molecules. In particular, the HNCO profiles are very similar to those of $\mathrm{CH}_{3} \mathrm{OH}, \mathrm{H}_{2} \mathrm{CO}, \mathrm{SO}$, and $\mathrm{SO}_{2}$, which are all shock tracers whose abundance has increased by more than a factor of 10 in the shocked gas with respect to the quiescent gas in L1157-mm. The HNCO line wings are narrower than SiO lines, in agreement with the findings of Zinchenko et al. (2000) in Galactic dense cores, but the $\mathrm{SiO}$ line profiles are particularly broad in the L1157 molecular outflow, being broader than those of all the other molecules.

As discussed by Bachiller \& Perez Gutierrez (1997), most of the molecules exhibit lines of similar intensity in B2 and B1. The exceptions are $\mathrm{CN}$, which is very intense towards $\mathrm{B} 1$ but not detected towards $\mathrm{B} 2$, and the sulfur-bearing molecules $\mathrm{SO}_{2}$ and OCS, whose lines are far more intense in B2 than in B1. We note that $\mathrm{HNCO}$, whose lines are a factor of 2 more intense in $\mathrm{B} 2$ than in B1, is indeed the only non-sulfur-bearing molecule showing this behavior. 
$\mathrm{mm}$

B1

B2

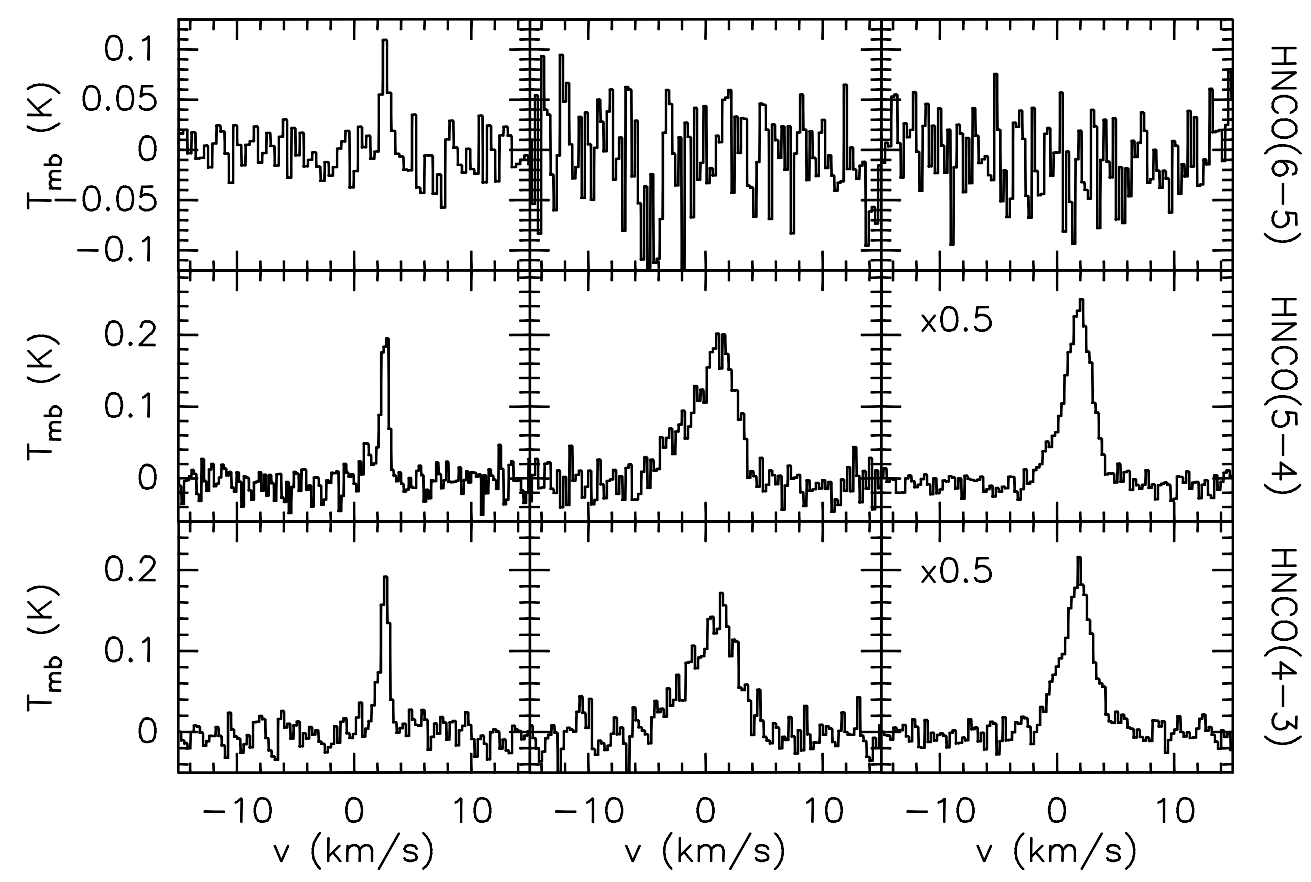

Fig. 2. Observed HNCO spectra. From bottom to top, the different panels show the HNCO $4_{04}-3_{03}, 5_{05}-4_{04}$, and $6_{06}-5_{05}$ spectra towards L1157 mm, $\mathrm{B} 1$, and $\mathrm{B} 2$.

\subsection{Enhanced HNCO abundances in the shocked gas}

The critical density of the HNCO transitions discussed here is high $\left((0.5-1) \times 10^{6} \mathrm{~cm}^{-3}\right.$; derived using the collisional coefficients in the LAMDA database for temperatures from $20 \mathrm{~K}$ to $320 \mathrm{~K}$, Schöier et al. 2005). Therefore, it is likely that most of the emission comes from dense and small clumps, such as those found in B1 with the IRAM Plateau de Bure interferometer (Benedettini et al. 2007; Codella et al. 2009). For instance, the angular size of the $\mathrm{CH}_{3} \mathrm{OH}$ clumps is $10^{\prime \prime}$. However, without high angular resolution observations it is difficult to assign a very precise value to the size of the source emitting in HNCO. Therefore, all the following results were obtained for two limiting cases. We first assumed that the size of the emitting region is $10^{\prime \prime}$ and computed the source brightness temperatures by taking into account the corresponding beam filling factor. Second, we assumed that the emitting region fills the primary beam of the telescope, and thus used $T_{\mathrm{mb}}$ temperatures.

\subsubsection{LTE analysis}

We computed HNCO column densities assuming optically thin emission and LTE excitation. We also computed the excitation temperatures of the $5_{05}$ and $4_{04}$ populations $\left(\mathrm{T}_{\mathrm{ex}}^{54}\right.$ ) and of the $6_{06}$ and $4_{04}$ populations $\left(\mathrm{T}_{\mathrm{ex}}^{64}\right)$. The temperatures $\mathrm{T}_{\mathrm{ex}}^{64}$ are very low (5-6 K for $\mathrm{mm}$ and $<6 \mathrm{~K}$ for B1 and B2). The excitation temperature $\mathrm{T}_{\mathrm{ex}}^{54}$ varies from 8 to $11 \mathrm{~K}$ for L1157-mm (depending on the assumptions made about the source size), while it varies from 9 to $19 \mathrm{~K}$ in $\mathrm{B} 1$ and $\mathrm{B} 2$ (see Table 3 ). To study with great precision the HNCO excitation, we require knowledge of more than three transitions. Here, to obtain a conservative estimation of the total column density of HNCO, we extrapolated the population in the $5_{05}$ and $4_{04}$ levels using the $\mathrm{T}_{\mathrm{ex}}^{54}$. The total HNCO column density would of course be higher, if the excitation temperature were actually lower. Using $\mathrm{T}_{\mathrm{ex}}^{54}$ and assuming LTE excitation, the total HNCO column densities are 0.2-0.8, 1-1.9, and 1.5-3.8 (in units of $10^{13} \mathrm{~cm}^{-2}$ ) for mm, B1, and B2, respectively. Since the excitation is similar in $\mathrm{B} 1$ and $\mathrm{B} 2$, the highest line intensities measured towards B2 translate into larger column densities, in contrast to the $\mathrm{CO}$ column density, which is a factor of 2 lower in B2 (Bachiller \& Perez Gutierrez 1997).

\subsubsection{RADEX analysis}

In addition, we studied the HNCO excitation using RADEX (van der Tak et al. 2007), which is a non-LTE excitation and radiative transfer code that decouples the statistical equilibrium and the radiative transfer equations using the escape probability method. The calculations assume lines of a given width and rectangular shape. Therefore, the variation in the optical depth over the profile is not taken into account. This is not a problem if the opacity of the lines is not very high. We used the HNCO$\mathrm{H}_{2}$ collisional coefficients listed in the LAMDA database (Schöier et al. 2005). The computations use a background temperature of $2.73 \mathrm{~K}$. We modeled the line fluxes (or integrated intensities) as a function of the hydrogen volume density $\left(n_{\mathrm{H}_{2}}\right)$, the HNCO column density $\left(N_{\mathrm{HNCO}}\right)$, the kinetic temperature $\left(T_{\mathrm{K}}\right)$, and the velocity dispersion. The range of hydrogen densities used is $10^{3}-$ $10^{6} \mathrm{~cm}^{-3}$ and that of $N_{\mathrm{HNCO}}$ is $10^{12}-10^{15} \mathrm{~cm}^{-2}$. Two kinetic temperatures were considered: a low kinetic temperature $(30 \mathrm{~K})$, only slightly higher than the excitation temperature derived with the HNCO lines and a much higher temperature $(250 \mathrm{~K})$, since the true gas temperature in the shocked gas may be considerably higher than the HNCO excitation temperatures. These temperatures probably cover the different physical conditions of L1157$\mathrm{mm}$ and the shocked sources B1 and B2. We computed models with velocity dispersions (linewidths) from 1 to $6 \mathrm{~km} \mathrm{~s}^{-1}$, which cover the measured range of linewidths in the three observed positions. For the range of physical conditions compatible with the observations, the opacity at the peak of the lines is low. Therefore, the predicted integrated intensities are independent 


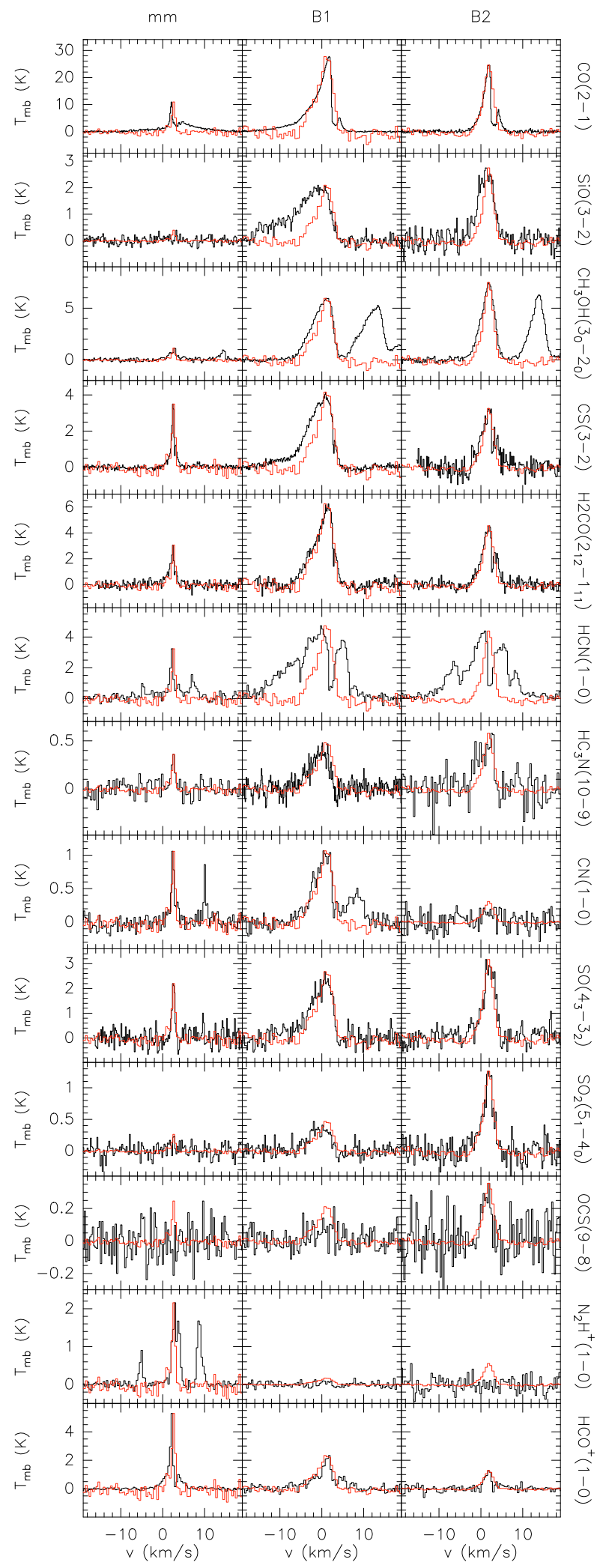

Fig. 3. Comparison of the $\mathrm{HNCO}\left(5_{05}-4_{04}\right)$ line profiles (in red) with the spectra of other species published by Bachiller \& Perez-Gutierrez (1997), in black lines. The HNCO peak temperature has been scaled to the peak temperature of the other molecule to compare profiles.

of the true linewidth used to compute the models. For instance, the figures discussed below were computed with a linewidth of $3 \mathrm{~km} \mathrm{~s}^{-1}$.
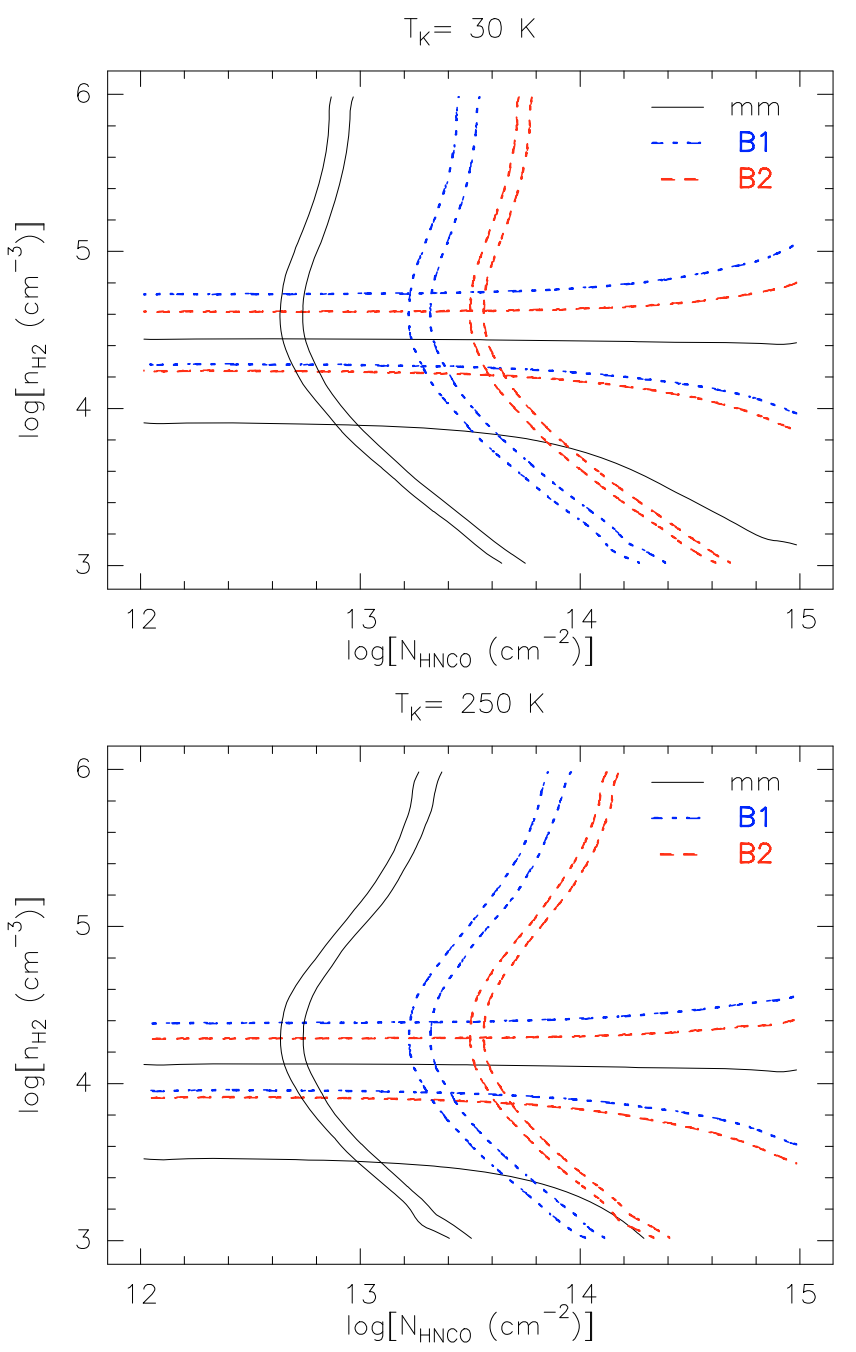

Fig. 4. RADEX results for the $5_{05}-4_{04}$ to $4_{04}-3_{03}$ flux ratio (horizontal curves) and the $4_{04}-3_{03}$ line flux (vertical curves) computed with a brightness temperature for a source size of $10^{\prime \prime}$ as a function of the hydrogen density, the HNCO column density and the kinetic temperature $(30 \mathrm{~K}$ in the upper panel and $250 \mathrm{~K}$ in the lower panel). A typical uncertainty of $10 \%$ in the line flux has been taken into account.

We based our analysis on the two lowest lines, which are detected towards the three observed positions. Figure 4 shows the model predictions for the $5_{05}-4_{04}$ to $4_{04}-3_{03}$ line ratio and the $4_{04}-3_{03}$ brightness temperature (for a source size of $10^{\prime \prime}$ ) as a function of $n_{\mathrm{H}_{2}}$ and $N_{\mathrm{HNCO}}$. The same diagrams for a source size equal to the telescope main beam (using $T_{\mathrm{mb}}$ ) are shown in Fig. 5.

The kinetic temperature cannot be constrained since equally good solutions can be found by just changing the hydrogen density by $\sim 0.5$ dex. For a given source size and kinetic temperature, the $\mathrm{H}_{2}$ density is similar for $\mathrm{B} 1$ and $\mathrm{B} 2$. The density varies from $10^{4}-10^{4.4} \mathrm{~cm}^{-3}$ for a source size of $10^{\prime \prime}$ and $T_{K}=250 \mathrm{~K}$, to $10^{4.6}-10^{5.2} \mathrm{~cm}^{-3}$ for sources filling the main beam and $T_{K}=30 \mathrm{~K}$. In L1157-mm, $n_{\mathrm{H}_{2}}$ is lower than that in B1 and B2 by $0.2-0.3$ dex.

The total HNCO columns densities are $0.2-1.3,0.6-2.5$, and 1.3-5.0 (in units of $10^{13} \mathrm{~cm}^{-2}$ ) for $\mathrm{mm}, \mathrm{B} 1$, and B2, respectively (Table 3). The lower limits are similar to those derived using LTE, while the upper limits for B1 and B2 are somewhat higher because opacity effects begin to play a role. Table 3 also shows HNCO abundances computed with the RADEX estimations of the 

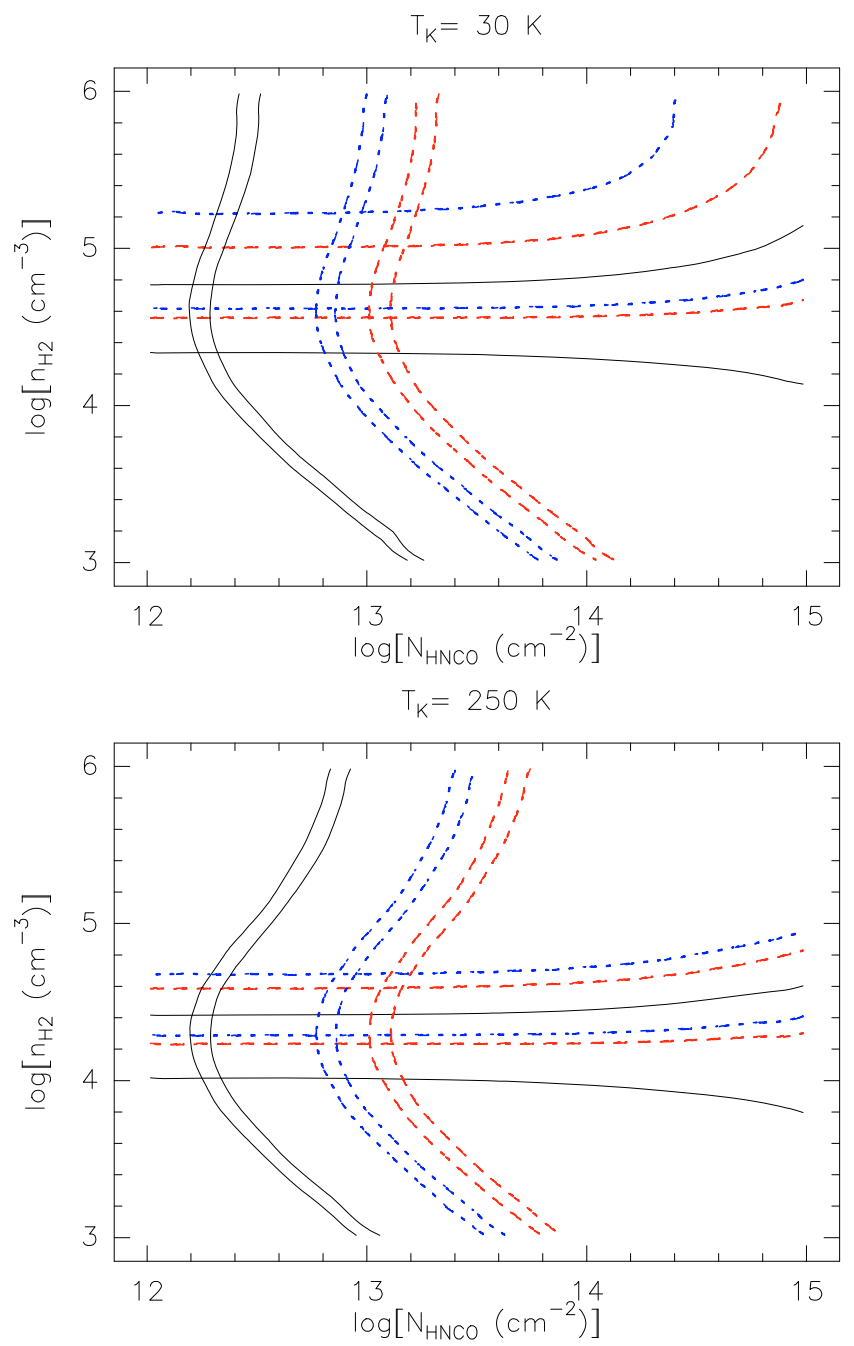

Fig. 5. Same than Fig. 4 but using $T_{\mathrm{mb}}$.

HNCO column density and the total $\mathrm{H}_{2}$ column density derived from CO by Bachiller \& Perez Gutierrez (1997). The HNCO abundances are $(0.3-1.2) \times 10^{-9}$ for L1157-mm, $(4.3-17.9) \times$ $10^{-9}$ for L1157-B1, and $(25-96) \times 10^{-9}$ for L1157-B2 (Table 3). Therefore, there is a clear increase in the HNCO abundance in the shocked regions, by a factor of 6-14 in B1 and by a factor of 34-83 in B2. This is the first direct evidence that HNCO is indeed a good tracer of shocked gas.

In the range of physical conditions and HNCO column densities derived from the $5_{05}-4_{04}$ and $4_{04}-3_{03}$ lines, the model predictions for the $6_{06}-5_{05}$ to $5_{05}-4_{04}$ ratio is $0.6-0.7$. In contrast, the measured ratio is $0.3-0.4$ for L1157- $\mathrm{mm}$ and lower than 0.3 for B1 and B2. As already mentioned above when discussing the LTE analysis, a multi-transition survey would be needed to fully understand the excitation of the molecule. In addition, HNCO maps with high angular resolution will be needed to determine the positions and sizes of the different HNCO clumps, and in particular to ascertain whether is any HNCO emission outside the IRAM-30 $\mathrm{m}$ beam at $2 \mathrm{~mm}$.

\subsubsection{Comparison with other Galactic sources}

Table 4 shows HNCO abundances with respect to $\mathrm{H}_{2}$ measured for other Galactic sources such as translucent clouds, photon-dominated regions (PDRs), dense cores, and hot cores.
Table 3. Results.

\begin{tabular}{lllll}
\hline \hline Source & $\begin{array}{l}T_{\mathrm{ex}}^{54 a} \\
\mathrm{~K}\end{array}$ & $\begin{array}{l}N_{\mathrm{HNCO}}{ }^{b} \\
10^{13} \mathrm{~cm}^{-2}\end{array}$ & $\begin{array}{l}N_{\mathrm{HNCO}}{ }^{c} \\
10^{13} \mathrm{~cm}^{-2}\end{array}$ & $\begin{array}{l}X(\mathrm{HNCO})^{d} \\
10^{-9}\end{array}$ \\
\hline $\mathrm{mm}$ & $8-11$ & $0.2-1.3$ & $0.2-1.3$ & $0.3-1.2$ \\
$\mathrm{~B} 1$ & $11-19$ & $0.6-2.5$ & $0.6-2.5$ & $4.3-17.9$ \\
$\mathrm{~B} 2$ & $9-16$ & $1.3-5.0$ & $1.3-5.0$ & $25-96$ \\
\hline
\end{tabular}

Notes. ${ }^{(a)}$ Excitation temperatures derived from the populations in the $5_{0,5}$ and $4_{0,4}$ levels. ${ }^{(b)}$ HNCO column density assuming LTE, optically thin lines and extrapolating using $T_{\mathrm{ex}}^{54} \cdot{ }^{(c)} \mathrm{HNCO}$ column density computed using RADEX. ${ }^{(d)} \mathrm{HNCO}$ abundance with respect to $\mathrm{H}_{2}$ (using the $\mathrm{H}_{2}$ column densities given by Bachiller \& Perez Gutierrez 1997).

Table 4. $\mathrm{HNCO}$ abundance with respect to $\mathrm{H}_{2}$ in different astronomical environments.

\begin{tabular}{lcc}
\hline \hline Source & $\begin{array}{c}X(\mathrm{HNCO}) \\
10^{-9}\end{array}$ & References \\
\hline L1157-B2 & $25-96$ & 1 \\
Translucent clouds & $0.2-5$ & 2 \\
Orion bar PDR & $<10^{-2}$ & 3 \\
Dense cores & $0.2-8.7$ & 4 \\
Orion Hot core & 5 & 5 \\
SgrB2M & 1 & 6 \\
W3(H 20$)$ & $5.0-27$ & 7 \\
G34.3+0.15 & 1.4 & 8 \\
IRAS 16293 & $0.17-4.0$ & 9,10 \\
GC clouds & $3-48$ & 11,12 \\
Starburst galaxies & $1-6.3$ & 13 \\
\hline
\end{tabular}

References. (1) This work; (2) Turner et al. (1999); (3) Jansen et al. (1995); (4) Zinchenko et al. (2000); (5) Blake et al. (1987); (6) Minh et al. (1998); (7) Helmich \& van Dishoeck (1997); (8) Bockelée-Morvan et al. (2000), using the HNCO column density given by MacDonald et al. (1996) and the $\mathrm{H}_{2}$ column density given by Hatchell et al. (1998); (9) van Dishoeck et al. (1995); (10) Bisschop et al. (2008); (11) Rizzo et al. (2000); (12) Martín et al. (2008), and references within; (13) Martín et al. (2009)

The highest HNCO abundance is found in hot cores and dense cores up to $\sim 8.7 \times 10^{-9}$. The HNCO abundance in L1157$\mathrm{mm}$ is similar to that measured in these dense and hot cores with the lowest HNCO abundance and in the only "hot corino" where the molecule has been previously detected, IRAS 16293 (van Dishoeck et al. 1995). The HNCO abundance in the L1157B1 shock is comparable, but can even be higher than that in hot cores and dense cores. For the L1157-B2 shock, the HNCO abundance is at least 4-11 times higher than that in hot cores and dense cores. The HNCO abundance in the L1157-B2 shock are indeed the highest HNCO abundances ever measured.

\section{Discussion}

\subsection{HNCO formation in molecular clouds and hot cores}

The possible formation pathways of HNCO in shocks have never been modeled. In contrast, HNCO has been included in some models of dark clouds and hot core chemistry. In the models by Iglesias (1977), HNCO is produced by the ion-neutral reaction $\mathrm{H}_{2}+\mathrm{HNCO}^{+} \rightarrow \mathrm{H}_{2} \mathrm{NCO}^{+}+\mathrm{H}$ followed by $\mathrm{H}_{2} \mathrm{NCO}^{+}+\mathrm{e}^{-} \rightarrow$ $\mathrm{HNCO}+\mathrm{H}$. Turner et al. (1999) considered three possible formation pathways, among which the only efficient one is the neutral-neutral reaction $\mathrm{CN}+\mathrm{O}_{2} \rightarrow \mathrm{NCO}+\mathrm{O}$ followed by $\mathrm{NCO}+\mathrm{H}_{2} \rightarrow \mathrm{HNCO}+\mathrm{H}$. The last reaction has an activation barrier that was estimated to be $\sim 1160 \mathrm{~K}$ by Turner et al. (1999) 
but could be as high as $4465 \mathrm{~K}$ (Tideswell et al. 2010). In any case, this reaction is not efficient at the typical temperature of a hot core $(\sim 200 \mathrm{~K})$. Therefore, gas phase chemistry alone cannot explain the HNCO abundances in hot cores. This is also the case for other species such as the complex organic molecules. Chemical models have consequently been developed to include reactions on the dust grain surface (Caselli et al. 1993; Garrod et al. 2008; Tideswell et al. 2010). In those works, hot cores are modeled in two stages.

In the first phase, a dark cloud of $n_{\mathrm{H}} \sim 10^{4} \mathrm{~cm}^{-3}$ undergoes an isothermal collapse. The collapse phase is halted once $n_{\mathrm{H}} \sim 10^{7} \mathrm{~cm}^{-3}$, which occurs after approximately $5 \times 10^{5}$ years (free-fall time). The typical temperature at this phase is 10$20 \mathrm{~K}$ and surface chemistry is very important then. Surface chemistry models (Garrod et al. 2008; Tideswell et al. 2010) produce $\mathrm{HNCO}$ as a secondary radical formed via the reaction $\mathrm{NH}+\mathrm{CO} \rightarrow \mathrm{HNCO} .{ }^{2}$ On the grain surface, $\mathrm{HNCO}$ can reach a maximum abundance of $10^{-5}$ (Tideswell et al. 2010) before being destroyed by new reactions with primary radicals $\left(\mathrm{H}, \mathrm{CH}_{3}\right.$, $\mathrm{HCO}, \mathrm{NH}, \ldots$ ) giving complex species as HNCHO, HNCOCHO, or $\mathrm{CH}_{3} \mathrm{CONH}$ (Garrod et al. 2008).

The cloud-collapse phase is followed by a warm-up phase in which the temperature increases to $\sim 200 \mathrm{~K}$. The timescale of the warm-up phase can be as short as $\sim 5 \times 10^{4} \mathrm{yr}$ in hot cores but can reach $10^{6} \mathrm{yr}$ in their low mass equivalent (hot corinos), giving a similar although slightly different chemistry (Garrod et al. 2008).

During the warm-up phase, molecules are evaporated from the grain mantles. With respect to HNCO, the models by Garrod et al. (2008) and Tideswell et al. (2010) indicate that its abundance increases monotonously with time in the gas phase, which implies that $\mathrm{HNCO}$ is not directly ejected from the grains. Instead, HNCO is formed by the destruction in the gas phase of the complex molecules formed from HNCO on the grain surface (Garrod et al. 2008; Tideswell et al. 2010). This mechanism can explain the HNCO abundances measured in hot cores of $10^{-9}$ $10^{-8}$. As already mentioned, the contribution of the Turner et al. (1999) and Iglesias (1977) formation pathways is negligible at the moderate temperature of a hot core.

The abundance of HNCO in L1157-mm is similar to that measured in the hot corino of the only other low mass protostar where HNCO has been observed (IRAS16293-2422, see Table 4). Therefore, the HNCO abundance measured in L1157$\mathrm{mm}$ can also be explained in the context of the hot-cores models discussed above. This would imply that L1157-mm can be considered as a hot corino, which is indeed in agreement with the intense emission of water detected by Herschel towards mm (Nisini et al., in prep). However, more observations will be needed to clearly establish the hot corino character of L1157$\mathrm{mm}$, in particular observations of complex organic molecules.

\subsection{HNCO formation in shocks}

What is the formation pathway of HNCO in the L1157 outflow shocks? The most likely scenario is a combination of grain surface and gas phase chemistry. In the context of shocks, molecules formed on the grain surfaces can be ejected to the gas phase due to grain sputtering instead of thermal evaporation

${ }^{2} \mathrm{HNCO}$ formation on the grain surfaces could also occur by means of reactions of $\mathrm{OCN}^{-}$with $\mathrm{NH}_{4}^{+}$or $\mathrm{H}_{3} \mathrm{O}^{+}$. The inverse reactions ( $\mathrm{HNCO}$ with $\mathrm{NH}_{3}$ or $\mathrm{H}_{2} \mathrm{O}$ ) are invoked to explain the presence of $\mathrm{OCN}^{-}$ices in the dust grains, (Demyk et al. 1998; van Broekhuizen et al. 2004). To our knowledge, these reactions have never been included in chemical models. as in hot cores. Therefore, the chemistry will be sensitive to the grain mantle composition at the time of the shock arrival. No models have yet been developed to study the HNCO abundance in dark clouds including grain surface chemistry. Dark cloud models by Tideswell et al. (2010) only consider gas phase reactions. Therefore, the exact grain mantle composition before the arrival of the shock in L1157-B1 and L1157-B2 is not known. Nevertheless, one can compare with the cloud-collapse phase of hot core models (the main difference is that the density during the collapse phase reaches higher values than in dark clouds). As discussed in the previous section, during the collapse phase the HNCO abundance on the grain surfaces reaches a peak of $10^{-5}$, grain sputtering at that time immediately producing a very high HNCO abundance in gas phase. Even the very high HNCO abundance in L1157-B2 $\left(\sim 10^{-7}\right)$ could be accounted for in this context.

On the other hand, gas phase chemistry in shocks differs considerably from that in hot cores because the temperature in the shocked gas can be much higher than that in a hot core. As already pointed out by Zinchenko et al. (2000), in shocks the reaction $\mathrm{NCO}+\mathrm{H}_{2}$ can be efficient in spite of the activation barrier. In addition, the reaction $\mathrm{CN}+\mathrm{O}_{2} \rightarrow \mathrm{NCO}+\mathrm{O}$ will be assisted by the high $\mathrm{O}_{2}$ abundances that are predicted in post-shock gas (Bergin et al. 1998; Gusdorf et al. 2008a,b). ${ }^{3}$

Using Tideswell et al. (2010) results, it is possible to verify whether gas phase chemistry alone could explain the HNCO abundance measured in the L1157 shocks. In one of their dark cloud models (DC1), they studied the HNCO formation exclusively via the gas phase neutral-neutral reactions of Turner et al. (1999) as a function of the rate coefficient for the reaction $\mathrm{NCO}+\mathrm{H}_{2} \rightarrow \mathrm{HNCO}+\mathrm{H}$. Their Fig. 1 shows that a rate coefficient $k$ higher than $10^{-14} \mathrm{~cm}^{3} \mathrm{~s}^{-1}$ is needed to explain the observed abundances in L1 157 of $10^{-8}-10^{-7}$. However, to produce a high $\mathrm{HNCO}$ abundance (a few $10^{-8}$ ) in a short time (several $10^{3} \mathrm{yr}$ ) the rate coefficient must be higher than $10^{-12} \mathrm{~cm}^{3} \mathrm{~s}^{-1}$. Taking into account the formula $k(T)=1.5 \times$ $10^{-11} \exp (-4465 / T) \mathrm{cm}^{-3} \mathrm{~s}^{-1}$ given by Tideswell et al. (2010), $k=10^{-12} \mathrm{~cm}^{3} \mathrm{~s}^{-1}$ implies a temperature higher than $1600 \mathrm{~K}$. In contrast, the minimum temperature would be $428 \mathrm{~K}$ if the activation barrier is only $1160 \mathrm{~K}$ (Turner et al. 1999). Gusdorf et al. (2008b) computed shock models to compare the modelled $\mathrm{SiO}$ and $\mathrm{H}_{2}$ emission to observations of L1157. The closest agreement between models and observations is found for a preshock density of $10^{4} \mathrm{~cm}^{-3}$ and a shock speed of $20 \mathrm{~km} \mathrm{~s}^{-1}$. In this shock, the gas temperature is higher than $430-1600 \mathrm{~K}$ for a few thousand years, which is consistent with both the dynamical age of the B1 and B2 shocks (2000-3000 yr, Gueth et al. 1998) and the chemical time necessary to produce a HNCO abundance of a few $10^{-8}$ only with gas phase reactions (Fig. 1 of Tideswell et al. 2010). Therefore, gas phase chemistry alone could explain the HNCO abundance in L1157-B1. However, it is unclear whether a HNCO abundance as high as that measured in L1157B2 $\left(\sim 10^{-7}\right)$ can be attained in a few thousand years only with gas phase reactions.

Therefore, we reckon that the most likely explanation of the high HNCO abundances in L1157 is dust grain mantles

\footnotetext{
3 This does not mean that the current $\mathrm{O}_{2}$ abundance in the L1157 shocks is high, but it may have been high just after the passage of a shock-wave as predicted by shock models. Since the chemistry in shocked regions proceeds rapidly, $\mathrm{O}_{2}$ can be rapidly converted into other species. Therefore, this is not inconsistent with the $\mathrm{O}_{2}$ abundance in the interstellar medium being found to be low $\left(<10^{-7}\right.$ Larsson et al. 2007).
} 
processing by the shock waves followed by neutral-neutral reactions in gas phase.

\subsection{Chemical differences between $B 1$ and $B 2$ and the role of the $\mathrm{O}_{2}$ chemistry}

We have discussed the line profiles and the line intensities of different species in Sect. 3.1. The HNCO profiles are very similar to those of $\mathrm{SO}$ and $\mathrm{SO}_{2}$. In addition, the lines of most of the molecules have similar intensities in $\mathrm{B} 1$ and $\mathrm{B} 2$ with the exception of $\mathrm{CN}$, which is more intense in $\mathrm{B} 1$, and $\mathrm{HNCO}, \mathrm{SO}_{2}$, and OCS, whose lines are more intense emission in $\mathrm{B} 2$ than in B1. These similarities between the HNCO and the sulfured molecules is somewhat puzzling.

The true reason for the chemical differences in B1 and B2 is not clear. The present gas density in B1 and B2 is similar (see Figs. 4 and 5 and Bachiller \& Perez Gutierrez 1997; Nisini et al. 2007). In contrast, based on the line profiles, the shock velocity may be higher in B1 than in B2. Taking into account the extreme $\mathrm{SiO}$ line wings, the difference could reach $10 \mathrm{~km} \mathrm{~s}^{-1}$, which according to the Gusdorf et al. (2008a) models could be significant.

Bachiller \& Perez Gutierrez (1997) suggested that the chemical differences between B1 and B2 could alternatively be due to different shock ages. In contrast to $\mathrm{SO}_{2}$ and OCS, the $\mathrm{H}_{2} \mathrm{~S}$ abundance is indeed lower in $\mathrm{B} 2$ than in $\mathrm{B} 1$. This is logical since $\mathrm{H}_{2} \mathrm{~S}$ is a parent molecule for other sulfured species. $\mathrm{H}_{2} \mathrm{~S}$ is formed on the grain surfaces and released to the gas phase by the effect of the shock waves. Other sulfur-bearing molecules such as $\mathrm{SO}$ and $\mathrm{SO}_{2}$ are produced in the gas phase very quickly (few $10^{3} \mathrm{yr}$ ) via reactions with $\mathrm{H}, \mathrm{OH}$, and $\mathrm{O}_{2}$ (Pineau des Forêts et al. 1993; Wakelam et al. 2005). One possible explanation of the differences between $\mathrm{B} 1$ and $\mathrm{B} 2$ is that the B2 shock could be older than that in $\mathrm{B} 1$ and that $\mathrm{H}_{2} \mathrm{~S}$ has been converted into $\mathrm{SO}$ and $\mathrm{SO}_{2}$. Even if there are uncertainties in the sulfur chemistry (see for instance Codella et al. 2005, and references therein), sulfur-bearing molecules such as $\mathrm{H}_{2} \mathrm{~S}, \mathrm{SO}, \mathrm{SO}_{2}, \mathrm{H}_{2} \mathrm{CS}$, and OCS have been invoked to be potential valuable probes of chemical evolution (Codella et al. 2005; Wakelam et al. 2005; Herpin et al. 2009). In particular, Wakelam et al. (2005) found that the $\mathrm{SO}_{2} / \mathrm{SO}$ ratio increases with the shock age. The higher $\mathrm{SO}_{2} / \mathrm{SO}$ ratio in $\mathrm{B} 2$ than in $\mathrm{B} 1$ is also indicative of an older shock in $\mathrm{B} 2$. Bachiller \& Perez Gutierrez (1997) also proposed that the different $\mathrm{CN}$ abundance in $\mathrm{B} 1$ and $\mathrm{B} 2$ can be accounted for in this scenario of an older shock in B2, since after an enhancement of $\mathrm{CN}$ in the shock, reactions such as $\mathrm{CN}+\mathrm{O} \rightarrow \mathrm{CO}+\mathrm{N}$ could be very efficient in destroying $\mathrm{CN}$. Our data globally agree with this scenario, nevertheless the clear anticorrelation of the $\mathrm{CN}$ and $\mathrm{HNCO}$ intensities and the similar abundances of $\mathrm{CN}$ and $\mathrm{HNCO}$ in L1157-B1 suggest that all the CN can indeed be transformed into $\mathrm{HNCO}$ once the shock has increased the temperature and the neutral-neutral reactions form $\mathrm{NCO}$ from $\mathrm{CN}$ and $\mathrm{O}_{2}$ and HNCO from NCO (see Sect. 4.2). Therefore, the chemical differences between $\mathrm{B} 1$ and B2 do support the scenario proposed in Sect. 4.2 to explain the HNCO abundances in the L1157 shocks.

In addition, the scheme proposed in Sect. 4.2 also provides insight into the possible link between $\mathrm{HNCO}$ and the sulfured molecules, in particular $\mathrm{SO}$ and $\mathrm{SO}_{2}$. In shocks, these molecules can be produced by the reactions $\mathrm{S}+\mathrm{O}_{2} \rightarrow \mathrm{SO}+\mathrm{O}$ and $\mathrm{SO}+\mathrm{OH} \rightarrow \mathrm{SO}_{2}+\mathrm{OH}$ (Pineau des Forêts et al. 1993; Charnley 1997; Wakelam et al. 2005). Therefore, the common link with $\mathrm{HNCO}$ would be formation pathways involving $\mathrm{O}_{2}$.

Another important shock tracer that could be linked to the $\mathrm{O}_{2}$ chemistry is SiO. Nevertheless, the situation regarding this molecule is more complex. First, there are models that can explain the $\mathrm{SiO}$ emission in shocks without a significant contribution of Si oxidation in gas phase, either by sputtering in gas-grain collisions if there is already $\mathrm{SiO}$ in the grain mantles (Gusdorf et al. 2008b) or by dust vaporization in grain-grain collisions (Guillet et al. 2009). Second, if one assumes that $\mathrm{SiO}$ is formed in gas phase, there is a threshold shock velocity of $\sim 25 \mathrm{~km} \mathrm{~s}^{-1}$ to eject $\mathrm{Si}$ from the grain cores by sputtering (Gusdorf et al. 2008a). In contrast, other molecules such as the organics are inside the grain mantles and there is no such high velocity threshold for them to be ejected into the gas phase. Therefore, if $\mathrm{SiO}$ comes directly from the grains (Gusdorf et al. 2008b; Guillet et al. 2009) or if it is formed in the gas phase (Gusdorf et al. 2008a), because of the shock velocity threshold, we do not expect to find similarities (and they are not observed) between the $\mathrm{SiO}$ emission and that of $\mathrm{SO}, \mathrm{SO}_{2}$, and $\mathrm{HNCO}$.

We note that the impressive $\mathrm{SiO}$ blue wing in $\mathrm{B} 1$ (Bachiller \& Perez Gutierrez 1997) is consistent with $\mathrm{SiO}$ formation having occurred, in comparison to other molecules, in the higher velocity gas. In this context, chemical differences between B1 and B2 may also be due to different shock velocities. If the shock velocity in $\mathrm{B} 2$ were actually lower than in $\mathrm{B} 1$ and the $\mathrm{SiO}$ formation less efficient, more $\mathrm{O}_{2}$ would be available in $\mathrm{B} 2$ to form $\mathrm{HNCO}$, $\mathrm{SO}$, and $\mathrm{SO}_{2}$.

\subsection{On the origin of the HNCO emission in galactic nuclei}

The HNCO abundance in the molecular clouds in the center of the Milky Way and in the nuclei of starburst galaxies is similar or a bit higher (at most by a factor of 2) than in Galactic hot cores (Table 4). HNCO becomes another piece in the well known puzzle of the chemistry of the Galactic center molecular clouds. This puzzle can be summarized as follows. The abundance of $\mathrm{SiO}$ and complex organic molecules in the Galacic center is as high as in hot cores (Martín-Pintado et al. 1997; Rodriguez-Fernandez et al. 2006; Requena-Torres et al. 2006). In contrast, (i) the emission in the Galactic center is extended over the central $300 \mathrm{pc}$ and does not resemble a collection of discrete sources with the size of a hot core $(\sim 0.1 \mathrm{pc})$; (ii) the gas density $\left(10^{3}-10^{4} \mathrm{~cm}^{-3}\right)$ is much lower than in hot cores; and (iii) the dust temperatures $(<30 \mathrm{~K})$ are also much lower than in hot cores. The Galactic center clouds present a "hot core chemistry without hot cores" (Requena-Torres et al. 2006). The origin of this chemistry is not known although it is understood to be triggered by some type of mechanical processes such as shock waves (Martín-Pintado et al. 1997, 2001; Rodríguez-Fernández et al. 2004). The origin of the shocks may be related to the complex dynamics in the inner regions of the Galaxy (Hüttemeister et al. 1998; RodriguezFernandez et al. 2006; Rodriguez-Fernandez \& Combes 2008).

Based on the spatial distribution of the $\mathrm{HNCO}$ and the comparison with other species, such as $\mathrm{CH}_{3} \mathrm{OH}$, Meier \& Turner (2005) also suggested that the HNCO emission in IC 342 traces shocks. Martín et al. $(2008,2009)$ also proposed that shocks may explain the high HNCO abundances measured in galactic nuclei.

Discussing the precise origin of shocks in Galactic nuclei is beyond the scope of this paper. Nevertheless, our observations support the scenario of HNCO tracing shocks in galactic nuclei since our L1157 results probe a medium of moderate $\mathrm{H}_{2}$ density, where the $\mathrm{HNCO}$ abundance is indeed high because of the grain processing and gas heating by shock waves. 


\section{Summary}

We have observed three lines of HNCO towards the protostar L1157 and its associated molecular outflow. Our aim has been to characterize the HNCO properties in shocked gas. HNCO was well detected in the shocked gas, where the abundance was found to increase by a factor of 6-34 with respect to the abundance in the protostar L1157- mm. The abundance in B1 and B2 is $(0.4-1.8) \times 10^{-8}$ and $(0.3-1) \times 10^{-7}$, respectively. The abundance in $\mathrm{B} 2$ is the highest ever measured, considerably higher than those in hot cores (a few $\left.10^{-9}\right)$ and galactic nuclei $\left(10^{-9}\right.$ $10^{-8}$ ). Our results demonstrate that the HNCO abundances measured in galactic nuclei can easily be attained in shocked gas, providing a solid basis to previous suggestions that the extended HNCO in galactic nuclei could trace large-scale shocks (Meier \& Turner 2005; Minh \& Irvine 2006).

The dominant formation pathway of $\mathrm{HNCO}$ in hot cores is grain mantle evaporation of complex molecules formed from HNCO and subsequent dissociation to produce again HNCO. In addition, there is contribution from gas phase reactions, but this is minor because of the high activation barriers of some reactions (Tideswell et al. 2010). In the L1157 shocks, we propose a relatively similar formation pathway, although with different relative importances of the dust surface and gas phase chemistry. First there should be grain mantle erosion by the shock waves that will increase the HNCO abundance in the gas phase. Second, in contrast to hot cores, the neutral-neutral reactions in the gas phase can be the dominant formation pathway due to the higher gas temperatures in the shocked gas with respect to the typical temperature of hot cores. These reactions start with the formation of $\mathrm{OCN}$ from $\mathrm{CN}$ and $\mathrm{O}_{2}$, which is expected to be very abundant in the gas phase of a magneto-hydrodynamic shock of relatively low velocity $\left(20-30 \mathrm{~km} \mathrm{~s}^{-1}\right)$ such as the L1157 shocks (Gusdorf et al. 2008a). The observed anticorrelation of $\mathrm{CN}$ and HNCO fluxes also support this scenario.

$\mathrm{HNCO}$ line profiles are very similar to those of $\mathrm{CH}_{3} \mathrm{OH}$ and $\mathrm{H}_{2} \mathrm{CO}$ lines, which is not surprising since all those molecules have, at least partially, a common origin linked to the grain surface chemistry and subsequent desorption. In addition, HNCO line profiles are also similar to some sulfur-bearing molecules such as $\mathrm{SO}$ and $\mathrm{SO}_{2}$. Another common property of $\mathrm{HNCO}$ and the sulfur-bearing molecules is that they are the only species that exhibit a more intense emission in $\mathrm{B} 2$ than $\mathrm{B} 1$. We propose that the common link between HNCO and the sulfured molecules is that in both cases there are formation pathways with reactions involving $\mathrm{O}_{2}$. HNCO and the sulfured molecules are reliable probes of chemical differentiation in shocks. In particular, in the case of L1157 the higher abundances in B2 than in B1 could be due to a lower shock velocity and/or an older shock in B2 than in $\mathrm{B} 1$.

Acknowledgements. N.J.R.-F. acknowledges fruitful discussions on chemistry and molecular outflows with S. Cabrit, A. Fuente and C. Codella. This work has been partially funded by the grant ANR-09-BLAN-0231-01 from the French Agence Nationale de la Recherche as part of the SCHISM project. We thank an anonymous referee for a very detailed report that helped to correct and clarify a number of issues in the original version of the manuscript.

\section{References}

Arce, H. G., Santiago-García, J., Jørgensen, J. K., Tafalla, M., \& Bachiller, R. 2008, ApJ, 681, L21

Bachiller, R., \& Perez Gutierrez, M. 1997, ApJ, 487, L93

Bachiller, R., Pérez Gutiérrez, M., Kumar, M. S. N., \& Tafalla, M. 2001, A\&A, 372,899
Benedettini, M., Viti, S., Codella, C., et al. 2007, MNRAS, 381, 1127 Bergin, E. A., Neufeld, D. A., \& Melnick, G. J. 1998, ApJ, 499, 777 Bisschop, S. E., Jørgensen, J. K., Bourke, T. L., Bottinelli, S., \& van Dishoeck, E. F. 2008, A\&A, 488, 959

Blake, G. A., Sutton, E. C., Masson, C. R., \& Phillips, T. G. 1987, ApJ, 315, 621 Bockelée-Morvan, D., Lis, D. C., Wink, J. E., et al. 2000, A\&A, 353, 1101 Caselli, P., Hasegawa, T. I., \& Herbst, E. 1993, ApJ, 408, 548 Charnley, S. B. 1997, ApJ, 481, 396

Churchwell, E., Wood, D., Myers, P. C., \& Myers, R. V. 1986, ApJ, 305, 405 Codella, C., Bachiller, R., Benedettini, M., et al. 2005, MNRAS, 361, 244 Codella, C., Benedettini, M., Beltrán, M. T., et al. 2009, A\&A, 507, L25 Dahmen, G., Huettemeister, S., Wilson, T. L., et al. 1997, A\&AS, 126, 197 Demyk, K., Dartois, E., D’Hendecourt, L., et al. 1998, A\&A, 339, 553 Garrod, R. T., Weaver, S. L. W., \& Herbst, E. 2008, ApJ, 682, 283

Gueth, F., Guilloteau, S., \& Bachiller, R. 1998, A\&A, 333, 287

Guillet, V., Jones, A. P., \& Pineau Des Forêts, G. 2009, A\&A, 497, 145

Gusdorf, A., Cabrit, S., Flower, D. R., \& Pineau Des Forêts, G. 2008a, A\&A, 482, 809

Gusdorf, A., Pineau Des Forêts, G., Cabrit, S., \& Flower, D. R. 2008b, A\&A, 490, 695

Hatchell, J., Thompson, M. A., Millar, T. J., \& MacDonald, G. H. 1998, A\&A, 338,713

Helmich, F. P., \& van Dishoeck, E. F. 1997, A\&AS, 124, 205

Herpin, F., Marseille, M., Wakelam, V., Bontemps, S., \& Lis, D. C. 2009, A\&A, 504,853

Hüttemeister, S. 1993, PhD Thesis, Dissertation, Bonn University

Hüttemeister, S., Dahmen, G., Mauersberger, R., et al. 1998, A\&A, 334, 646

Iglesias, E. 1977, ApJ, 218, 697

Jackson, J. M., Armstrong, J. T., \& Barrett, A. H. 1984, ApJ, 280, 608

Jansen, D. J., Spaans, M., Hogerheijde, M. R., \& van Dishoeck, E. F. 1995, A\&A, 303, 541

Kuan, Y., \& Snyder, L. E. 1996, ApJ, 470, 981

Larsson, B., Liseau, R., Pagani, L., et al. 2007, A\&A, 466, 999

Lindqvist, M., Sandqvist, A., Winnberg, A., Johansson, L. E. B., \& Nyman, L. 1995, A\&AS, 113, 257

MacDonald, G. H., Gibb, A. G., Habing, R. J., \& Millar, T. J. 1996, A\&AS, 119, 333

Marcelino, N., Cernicharo, J., Tercero, B., \& Roueff, E. 2009, ApJ, 690, L27

Martín-Pintado, J., de Vicente, P., Fuente, A., \& Planesas, P. 1997, ApJ, 482, L45

Martín-Pintado, J., Rizzo, J. R., de Vicente, P., Rodríguez-Fernández, N. J., \& Fuente, A. 2001, ApJ, 548, L65

Martín, S., Requena-Torres, M. A., Martín-Pintado, J., \& Mauersberger, R. 2008, ApJ, 678, 245

Martín, S., Martín-Pintado, J., \& Mauersberger, R. 2009, ApJ, 694, 610

Mauersberger, R., Guelin, M., Martin-Pintado, J., et al. 1989, A\&AS, 79, 217

Meier, D. S., \& Turner, J. L. 2005, ApJ, 618, 259

Minh, Y. C., \& Irvine, W. M. 2006, New Astron., 11, 594

Minh, Y. C., Haikala, L., Hjalmarson, A., \& Irvine, W. M. 1998, ApJ, 498, 261

Minh, Y. C., Kim, S., Pak, S., et al. 2005, New Astron., 10, 425

Nguyen-Q-Rieu, Henkel, C., Jackson, J. M., \& Mauersberger, R. 1991, A\&A, 241, L33

Nisini, B., Codella, C., Giannini, T., et al. 2007, A\&A, 462, 163

Pineau des Forêts, G., Roueff, E., Schilke, P., \& Flower, D. R. 1993, MNRAS, 262,915

Requena-Torres, M. A., Martín-Pintado, J., Rodríguez-Franco, A., et al. 2006, A\&A, 455, 971

Rizzo, D., Hüttemeister, S., \& Dahmen, G. 2000, in Molecules in Space and in the Laboratory, ed. I. Porceddu \& S. Aiello, 141

Rodriguez-Fernandez, N. J., \& Combes, F. 2008, A\&A, 489, 115

Rodríguez-Fernández, N. J., Martín-Pintado, J., Fuente, A., \& Wilson, T. L. 2004, A\&A, 427, 217

Rodriguez-Fernandez, N. J., Combes, F., Martin-Pintado, J., Wilson, T. L., \& Apponi, A. 2006, A\&A, 455, 963

Schöier, F. L., van der Tak, F. F. S., van Dishoeck, E. F., \& Black, J. H. 2005, A\&A, 432, 369

Snyder, L. E., \& Buhl, D. 1972, ApJ, 177, 619

Tideswell, D. M., Fuller, G. A., Millar, T. J., \& Markwick, A. J. 2010, A\&A, 510, A85

Turner, B. E., Terzieva, R., \& Herbst, E. 1999, ApJ, 518, 699

van Broekhuizen, F. A., Keane, J. V., \& Schutte, W. A. 2004, A\&A, 415, 425

van der Tak, F. F. S., Black, J. H., Schöier, F. L., Jansen, D. J., \& van Dishoeck, E. F. 2007, A\&A, 468, 627

van Dishoeck, E. F., Blake, G. A., Jansen, D. J., \& Groesbeck, T. D. 1995, ApJ, 447,760

Wakelam, V., Ceccarelli, C., Castets, A., et al. 2005, A\&A, 437, 149

Zinchenko, I., Henkel, C., \& Mao, R. Q. 2000, A\&A, 361, 1079 